\title{
6 Diskussion
}

Im folgenden Kapitel ordne ich die Forschungsergebnisse entlang relevanter wissenschaftlicher Diskussionen ein. Für die Untersuchung des komplexen Themas erwies sich ein theoretisch interdisziplinärer und methodisch explorativer Ansatz als notwendig. In der zusammenfassenden Diskussion zentraler Befunde ergänze ich daher die bisher vor allem medizinsoziologischen und medizinethischen Rahmungen des Themas um eine psychologische Perspektive ärztlichen Erlebens und Handelns in der Behandlung schwerstkranker und sterbender Patient*innen im Krankenhaus (vgl. Kap. 6.1.). In einer methodologischen Betrachtung prüfe ich die Stärken und die Limitation der Arbeit (vgl. Kap. 6.2.) und leite abschließend in einem Ausblick Perspektiven für Forschung und Praxis ab (vgl. Kap. 6.3.).

\subsection{Einordnung der Untersuchungsergebnisse}

Ziel dieser Arbeit war es, die gegenwärtige Behandlungspraxis schwerstkranker und sterbender Patient`innen im Krankenhaus jenseits einer spezialisierten palliativmedizinischen Versorgung zu explorieren. Für den quantitativ relevantesten Versorgungsbereich dieser Patient*innengruppe zeigt sich in der Forschung ein Desiderat. Ausgehend von der Hypothese, dass Ärztinnen im arbeitsteiligen und hierarchischen System Krankenhaus eine Schlüsselposition einnehmen, stehen ihre Erfahrungen im Zentrum der Untersuchung. Es zeigte sich, dass ärztliche Entscheidungen insgesamt in der medizinischen Behandlung und damit auch bei Schwerstkranken und Sterbenden eine zentrale Rolle spielen, was zugespitzt in der Schlüsselkategorie des ärztlichen Postulats des Sterbendürfens im Krankenhaus sichtbar wird. Daher werde ich zunächst die Einflüsse, Arbeitsaufträge und das Belastungserleben im ärztlichen Entscheidungsprozess zusammenfassen (vgl. Kap. 6.1.1.), um dann die Bedeutung des Postulats für Theorie und Praxis zu diskutieren (vgl. Kap. 6.1.2.). Die Ergebnisse verweisen zudem auf die gegenwärtige Dualität von Akut- und Palliativmedizin (vgl. Kap. 6.1.3.) sowie vielfach auf eine Fehlversorgung Sterbender im Krankenhaus (vgl. Kap. 6.1.4.). Mit der Einordnung der Befunde in die aktuelle wissenschaftliche Diskussion lassen sich die Präkonzepte aus Kapitel $3 \mathrm{zu}$ neuen Hypothesen weiterentwickeln, die ich durch eine Rahmung kenntlich mache.

\subsubsection{Sterben im Krankenhaus als ärztliche Entscheidung - Behandlungsaufträge, Einflussfaktoren und Belastungserleben}

Das ärztliche Behandlungserleben von Schwerstkranken und Sterbenden im Krankenhaus lässt sich auf das Thema Behandlungsentscheidungen als zentrale ärztliche Tätigkeit zuspitzen. Die zu treffenden Entscheidungen haben eine Spannbreite von 
diagnostischen Maßnahmen bis hin zu Entscheidungen am Lebensende (EoL). Im Kontext der vorliegenden Studie wurde das Thema Entscheidungsfindung von den IP vor allem als EoL-Entscheidung diskutiert. Gerade die prognostische Unsicherheit bei Schwerstkranken, d. h. potentiell Sterbenden, liefert einen breiten Interpretationsspielraum mit vielfach diskrepanten Einschätzungen der Beteiligten. Wenngleich sich Vogds Hinweis bestätigt hat, dass sich nicht nur im medizinischen, sondern auch im ärztlichen Handeln Routinen und Normen entwickeln [13, S. 394], erscheint aufgrund der Ergebnisse der vorliegenden Studie die von Wettreck eingeführte Differenzierung von ärztlichem und medizinischem Handeln durchaus hilfreich [252]. In beiden untersuchten Kliniken sind Stationsärzt*innen mehrheitlich Assistenzärzt*innen und in fachärztlicher Ausbildung. Bei diesen klinischen Novizen zeigt sich die Differenz zwischen dem ärztlichen Handeln im präsentierten Anspruch und der noch suchenden Bewegung nach einem routinierten medizinischen Handeln [159, S. 73].

Vogd fragte in seiner Untersuchung $\mathrm{zu}$ ärztlichen Entscheidungsprozessen im Spannungsfeld von System- und Zweckrationalität, warum die Frage des Sterbens überhaupt als medizinisches Entscheidungsproblem verhandelt wird [13, S. 349f]. Aus soziologischer Perspektive sieht er in der medizinischen Rahmung des medizinisch nicht zu lösenden Problems Tod die ärztliche Handlungsfähigkeit durch neue Entscheidungssituationen entsprechend der institutionellen Logik eines Krankenhauses wiederhergestellt. Die Ergebnisse der vorliegenden Unterstützung bestätigen Vogds Ergebnisse dahingehend, dass erst im Anschluss an eine Entscheidung über das Sterben eine (dann vor allem pflegerische) Sterbebegleitung erfolgt. Hier lässt sich eine Professionalisierungstendenz erkennen. Denn mit der Festlegung, auch das Sterben im Krankenhaus als ärztliche Entscheidung einzuordnen, erfolgt eine professionelle Ermächtigung über einen Tätigkeitsbereich, der bisher wenig anerkannt ist. Das Ergebnis kann allerdings auch gegenteilig interpretiert werden: als ein In-die-Pflicht-Nehmen der Organisation und damit als „technokratische Regression“ [382, S. 45].

Sterbebegleitung wird nur selten als medizinischer (z. B. im Rahmen von Symptombehandlung) und kaum als ärztlicher Auftrag (z. B. Aufklärung der Angehörigen über eine Sterbesituation) angesehen, sondern vorrangig im Aufgabenbereich der Pflege verortet. Führt das zur Stärkung der pflegerischen Position im Krankenhaus? Oder ,[s]ind Medizin und Pflege so unvereinbar, dass sie regelrecht getrennt betrachtet werden müssen?“, wie der Medizinanthropologe Arthur Kleinman in seinen Überlegungen zur Rolle der Pflege im Gesundheitswesen fragt [127, S. 163]. Wie die vorliegenden Ergebnisse zeigen, dominieren ärztliche Entscheidungen in der interprofessionellen Hierarchie mit Ärztinnen an der Spitze das routinierte Nebeneinander der pflegerischen und ärztlichen Professionen im Krankenhaus. Konflikte im Zusammenhang mit Behandlungsentscheidungen bei Sterbenden werden interdisziplinär, intradisziplinär und/oder interprofessionell als „Dauerklassiker“ zwischen Ärzt*innen und Pflegenden beschrieben (vgl. Kap. 5.2.1.2.). So werden Pflegende zwar als hoch kompetent in der Patient`innenbeobachtung und geradezu beauftragt mit der Sterbebegleitung und Unterstützung der Angehörigen gesehen, als Entscheidungsträger 
aber nicht. Gleichwohl wird ihnen im Erkennen einer verschlechterten Erkrankungssituation und in der Einschätzung von Sterbeprozessen eine hohe fachliche Kompetenz sowie von allen IP - unabhängig von ihrer Berufserfahrung oder hierarchischen Position - mehr Erfahrung zugeschrieben (vgl. Kap. 5.2.1.3.).

Werden Patient*innen ärztlich als Sterbende definiert, erfolgt ein Aufgabenwechsel hin zur vorrangig pflegerischen Versorgung. Gelingt der Wechsel, halten die IP eine würdevolle Behandlung, d. h. Pflege, auch im Krankenhaus für möglich. Doch diese Kompetenzzuschreibung hat keine Auswirkung auf eine veränderte hierarchische Position oder Rolle der Pflege im Entscheidungsprozess (vgl. Kap. 5.2.1.3.). Die IP beschreiben durchaus eine moralische Verpflichtung, die Ärzt*in-Patient*in-Beziehung bei Sterbenden aufrechtzuerhalten. Streckeisen hat im Umgang mit Schwerstkranken und Sterbenden eine Bedrohung der ärztlichen Rolle im Gegensatz zur pflegerischen Rolle identifiziert [110, S. 198]. In diesem Sinne verweisen die Befunde zur Übergabe des Behandlungsauftrages bei Sterbenden an Pflegende auf die Aktualität von Parsons Rollenkonzeption [180].

Der insgesamt fehlende organisatorische Behandlungsauftrag eines Krankenhauses für Sterbende mit der Konsequenz einer nicht ausreichend ökonomischen Berücksichtigung wird bisher entweder individuell oder in Ausnahmen abteilungsphilosophisch mit einer vorrangig ethisch-moralischen und habituellen Begründung aufgefangen. Mit den dargestellten Idealen für die Behandlung Sterbender zeigen sich Ärztinnen und Ärzte als soziale Repräsentanten einer ethisch-moralisch geführten gesellschaftlichen Diskussion.

Anders als im Präkonzept formuliert, lässt sich in den Daten primär keine ärztliche Verunsicherung über den medizinischen Behandlungsauftrag bei Sterbenden erkennen, sobald sie als Sterbende definiert werden. Die ärztliche Unsicherheit bezieht sich auf den davor liegenden Definitions- und Entscheidungsprozess mit einer Zentrierung auf die Frage nach dem Zeitpunkt des Sterbens. Die konkrete Sterbebegleitung, vor allem mit dem Attribut einer würdevollen Behandlung, wird als pflegerische Aufgabe angesehen.

Die vielfältigen Einflüsse auf Entscheidungsprozesse, daraus resultierende Behandlungsaufträge und mögliche Belastungen wurden im Hinblick auf die psychische und soziale Situation der Ärzt^innen analysiert. Dabei stellt sich die hierarchische Position als zentraler Einflussfaktor dar. Je höher die ärztlich-hierarchische Position, desto ferner von den Patient*innen ist die ärztliche Tätigkeit und desto mehr Verantwortung übernehmen die Ärztinnen im Entscheidungsprozess. In der untersuchten Universitätsklinik ist diese Arbeitsteilung als deutlich steilere Hierarchie ausgeprägt. Das hierarchische Erleben unterscheidet sich nicht nur zwischen den untersuchten Kliniken, sondern auch zwischen den medizinischen Fachdisziplinen und Funktionsbereichen mit unterschiedlichen Kulturen. Die Ergebnisse zeigen, dass sich die Stationsärzt*innen zwar vorrangig als „Ausführende“ innerhalb der ärztlichen Hierarchie erleben, gleichzeitig aber auch als Vermittler zwischen Patientinnen-, Angehörigen-, Pflegen- 
den-, ärztlichen und Organisationsinteressen [13, S. 363]. Dieser stationsärztliche Anspruch spiegelt sich als Auftrag im Konsensideal wider. Konsens wird weniger als geteilte Entscheidungsfindung dargestellt, denn als Übermittlung einer hierarchisch getroffenen ärztlichen Entscheidung an andere Akteure in der Behandlung, wie ärztliche Kolleg^innen, Pflegende, Patient*innen oder Angehörige, verbunden mit dem Wunsch, diese mögen die Entscheidung annehmen und gutheißen (vgl. Kap. 5.2.3.).

Das Konsensideal steht in keinem Widerspruch zur deutlich formulierten und für notwendig erachteten Entscheidungshierarchie in der Behandler*innenpraxis (vgl. Kap. 5.2.1.). Denn grundsätzlich wird eine Hierarchie von den vorrangig stationsärztlichen IP als entlastend erlebt, vor allem, wenn komplexe, uneindeutige und unangenehme Entscheidungen zu treffen sind (z. B. bei differenten Wünschen von Patient*innen/Angehörigen und in EoL-Situationen). Dieser Befund lässt sich mit ihrer Position als Novizen mit geringer beruflicher Erfahrung erklären. Unzufriedenheit mit einer hierarchisch getroffenen Entscheidung entsteht erst, wenn die Einschätzung der Erkrankungssituation nicht geteilt wird; oder aber, wenn eine Entscheidung hierarchisch nicht getroffen wird (vgl. Kap. 5.2.1.2. \& Kap. 5.2.1.1.). In beiden Fällen kann es in der Konfrontation mit Sterbeprozessen zu ethisch-moralischen Konfliktlagen und Behandlungsdilemmata bei den Stationsärzt*innen im Sinne eines breiter gefassten Moral Distress (MD) kommen. Es ist also nicht unbedingt so, dass sie wissen, wie sie handeln würden und es nur nicht dürfen [264]. Bereits die Verwicklung in eine moralisch unerwünschte Situation - bei fehlender Entscheidung oder nicht konsensuell getroffener Entscheidung - führt zu psychischen Reaktionen [273, 383]. Psychisch belastend und möglicherweise zum Dilemma wird eine Sterbesituation auch dann, wenn quälende Bilder (schreckliches Symptomerleben, protrahiertes Sterben und ein unerwarteter Krankheitsverlauf) auftreten und diese das ärztliche Handeln im Kontakt mit diesen Patient*innen verunsichern (vgl. Kap. 5.1.5.2.2.).

Die Ergebnisse bestätigen die für den Kontext Akutkrankenhaus vielfältig beschriebene „Kampf-Kultur“ [252, S. 47; 209, S. e131] auch für palliative und Sterbesituationen. Der Kampf um Lebensrettung bei Sterbenden ist damit ein möglicher Ausgangspunkt für ethische Dilemmata [253] und psychische Belastung. In der Konfrontation mit Sterbenden, Schwerstkranken sowie Langliegern erscheint es für die interviewten Ärzt^innen deutlich schwieriger, sich aus der persönlichen Beziehung zu lösen sowie die eigene fachliche und emotionale Überforderung und Hilflosigkeit bis hin zu Todesängsten und medizinischen Misserfolgen auszublenden. Damit werden Befunde im Zusammenhang mit der Behandlung schwerstkranker und sterbender Patient*innen bestätigt [91, 106, 116, 255-256, 259]. Da MD und Burnout in den Interviews nicht regelhaft erfragt wurden, haben diese Überlegungen hypothetischen Charakter.

Diverse Untersuchungen zur ärztlichen Kommunikation und Entscheidungsfindung bei Schwerstkranken und Sterbenden, meist im Kontext einer onkologischen oder intensivmedizinischen Behandlung, haben Einflussfaktoren beschrieben [156, 200, 209, 211, 384-385]. Besonders differenziert zeigen sich die Ergebnisse von Gra- 
nek et al. [209], die in ihrer Untersuchung der onkologischen Behandlung in drei kanadischen Krankenhäusern verschiedene Einflussfaktoren gefunden haben, die eine frühzeitige und ehrliche Aufklärung verhindern. Diese lassen sich drei Bereichen zuordnen: dem ärztlichen, dem der Patient^innen und dem institutionellen. Alle dort benannten Einflüsse wurden in der vorliegenden Studie nicht nur für Aufklärungssituationen, sondern auch für zuverlässige ärztliche Therapieentscheidungen in allen untersuchten medizinischen Fachdisziplinen bestätigt (vgl. Kap. 5.2.). Für den ärztlichen Bereich benennen Granek et al. folgende Einflussfaktoren [209, S. e131]: Unkenntnisse über eine palliative Behandlung, ein persönliches Unbehagen gegenüber Tod und Sterben, eine diffuse Zuordnung von Verantwortlichkeit unter den Kolleg*innen, einen Dem-Tode-trotzenden-Modus (,death-defying mode“), einen Mangel an Erfahrung sowie eine fehlende Anleitung.

Die Ergebnisse der vorliegenden Untersuchung verweisen mit Blick auf die „diffuse Verantwortlichkeit“ insbesondere auf eine starke Personengebundenheit von Entscheidungen bei einer hierarchischen Verantwortungsübernahme mit einem (ober)ärztlichen Entscheidungsspektrum von „es bis zum Äußersten zu treiben“ bis „machen wir nichts mehr“ (vgl. Kap. 5.2.1.1.). Benannten Granek und Kolleg^innen als Einflussfaktoren aus dem Patient ${ }^{\star}$ innenbereich Patient`innen und/oder Angehörige, die nicht gerne über das Lebensende sprechen, Sprachbarrieren und jüngeres Alter [209, S. e132], zeigen sich in meiner Untersuchung Ergänzungen zum Einfluss des Alters: Nicht nur junge, sondern auch alte Menschen als Patient*innen verändern die Entscheidungssituation insbesondere bei EoL-Entscheidungen. Zudem haben die Sichtbarkeit von Symptomen (vgl. Kap. 5.2.5.2.) und die Ansprechbarkeit und Bekanntheit der Patient*innen (vgl. Kap. 5.2.5.1.) einen Einfluss auf die Entscheidungsfindung. Sprachbarrieren wurden von keinem der interviewten Ärzt*innen thematisiert. Nur ein Arzt wies auf kulturelle Unterschiede im Umgang mit Trauer und Verlust hin, die zu diskrepanten Vorstellungen von Angehörigen über eine Erkrankungs- und Behandlungssituation führen können (vgl. Kap. 5.2.5.3.). Die fehlende ärztliche Thematisierung des kulturellen Hintergrunds und/oder der Sprachdefizite von Patient`innen und/oder Angehörigen sind vor dem Hintergrund der Häufigkeit von Menschen mit Migrationshintergrund ${ }^{52}$ zumindest in der untersuchten Universitätsklinik in einer deutschen Großstadt bemerkenswert.

Schildmann und Kolleg^innen haben bereits für ärztliche Mitglieder der DGP [249] und für hämatologisch/onkologisch tätige Ärztinnen [250, 390] vor allem nicht-medizinische Faktoren als Kriterien für Behandlungsentscheidungen heraus-

52 Zu Beginn der Untersuchungszeitraumes 2013 hatten 20 \% der deutschen Bevölkerung einen Migrationshintergrund, in Berlin waren es mehr als 24 \% [386]. Das Statistische Bundesamt definiert Migrationshintergund wie folgt: „Eine Person hat dann einen Migrationshintergrund, wenn sie selbst oder mindestens ein Elternteil nicht mit deutscher Staatsangehörigkeit geboren ist.“ [387, S. 7]. Die nach wie vor inadäquate Gesundheitsversorgung dieser sehr heterogenen Gruppe ist inzwischen auch für die Palliativversorgung beschrieben worden [388-389]. 
gestellt. Daran anschließend haben sie die notwendige Explizierung individueller ärztlicher Werte für ethisch fundierte Entscheidungen formuliert. Diese Forderung ist auf Grundlage der vorliegenden Befunde zu kulturellem Lernen und Lehren in der Medizin, mit deutlicher Bezugnahme auf persönliche moralische Werte und Erfahrungen von Ärztinnen unterschiedlichster medizinischer Fachdisziplinen, aufrechtzuerhalten.

Als Faktoren aus dem institutionellen Bereich haben Granek und Kolleg*innen ein Stigma in Bezug auf Palliative Care, das Fehlen eines klaren Protokolls bei EoLFragen sowie mangelnde Schulung zur Gesprächsführung analysiert [209, S. e132]. Für den vorliegenden Untersuchungskontext lassen sich alle benannten Einflussfaktoren bestätigen. Bezogen auf Entscheidungssituationen berichten die IP für den Intensivbereich durchaus von Standard Operating Procedures (SOP) als standardisierte Entscheidungen (z. B. bei Aufnahmen). Das berichtete Prozedere richtet sich nicht auf die Aufklärung von Patient`innen und/oder Angehörigen, sondern dient der intraprofessionellen Klärung einer medizinischen Indikation für eine ITS-Verlegung [248]. Hier wird ein Medical-Futility-Ansatz im Entscheidungsprozess sichtbar, den die interviewten Ärzt*innen für Palliativpatient`innen und bei Vorliegen einer PV oftmals - ungerechtfertigt - als restriktiv erleben (vgl. Kap. 5.3.3.2.). Bezugnehmend auf die Palliativ-Dimension wird deutlich, dass 'palliativ' von den IP nicht mit 'sterbend' assoziiert wird, weshalb auch eine intensivmedizinische Behandlung palliativer Patient^innen in akuten Situationen gerechtfertigt erscheint.

Im Hinblick auf die Kommunikation über Diagnose, Prognose und Therapie bestätigen die Ergebnisse sowohl die zentrale Bedeutung der Ärzt*in-Patient*in-Beziehung in aller Vielfältigkeit [170, 185, 203-205] als auch die zentrale ärztliche Rolle bei medizinischen Entscheidungen [192]. Insbesondere die ärztliche Rahmung der Behandlung von Schwerstkranken und Sterbenden als komplexe und prekäre medizinische Problemlage [13, S. 345] bringt Ärztinnen einerseits in die Experten-Rolle und andererseits in das Dilemma divergierender Wünsche [247]. Ob die Patient*innen im Entscheidungsprozess der ärztlichen Expertise vertrauen oder ihre Autonomie bei Entscheidungen betonen [200-201] wird von den Ärztinnen als gleichermaßen belastend erlebt, wenn die Therapieentscheidung unter (prognostischer) Ungewissheit erfolgt, wenn eine hierarchische Entscheidung nicht der eigenen Einschätzung entspricht bzw. wenn eine hierarchische Entscheidung trotz veränderter Erkrankungssituation ausbleibt oder wenn die Patient ${ }^{\star}$ innenentscheidung nicht mit der ärztlichen Behandlungsperspektive übereinstimmt [246]. In der Vielfalt der Einflüsse auf Therapieentscheidungen, die in Kapitel 5.2. dargestellt sind, wird zum einen das komplexe Geschehen und zum anderen der habituelle ärztliche Einfluss offenkundig. Der „scholastische Irrtum vom rationalen Entscheiden“ zeigt sich damit einmal mehr [13; 225, S. 176].

Interessant ist, dass in den Studienergebnissen der medizinethisch und berufspolitisch postulierte Wandel von der Fürsorge zur Autonomie, den ich in Kapitel 2.1.2. skizziert habe, kaum sichtbar wird. Besonders deutlich wird das in den vier 
Aufklärungstypen, die ich für die Ärzt*in-Patientin-Kommunikation rekonstruieren konnte: eine vermeidende, eine zurückhaltende, eine Heilung visionierende und eine offene ärztliche Aufklärung (vgl. Kap. 5.2.4.). Auf die Diskussion um Shared Decision Making (SDM) Bezug nehmend, muss konstatiert werden, dass sich wenig vom partizipativen Beteiligungsgedanken [188] in den im untersuchten Forschungsfeld rekonstruierten Aufklärungsstilen findet. Selbst der Typus eines offenen Aufklärungsstils (vgl. Kap. 5.2.4.) verweist nicht auf eine Beteiligung von Patient^innen, sondern vielmehr auf das „Paradigma des bewussten Sterbens“, welches Saake et al. in ihrer Untersuchung auf Palliativstationen rekonstruiert haben [5]. Gleichwohl verweisen alle vorgefundenen ärztlichen Ideale, allen voran das Konsensideal, im Kontakt mit Sterbenden auf beziehungsethische Überlegungen [28, S. 63ff].

Deutlich wird der fehlende Wandel vom Fürsorge- zum Autonomieprinzip, der 2009 juristisch im Patient*innenverfügungsgesetz verankert wurde, auch in der beschriebenen fehlenden Berücksichtigung von PV oder des mündlich formulierten Patient^innenwillens. Die Ergebnisse zeigen, dass PV gerade bei älteren und onkologisch Erkrankten zwar häufiger vorliegen, deren Inhalte allerdings von den IP als oftmals nicht zutreffend für die aktuelle Erkrankungssituation eingeschätzt werden (vgl. Kap. 5.2.6.). Damit bestätigen sich Untersuchungsergebnisse von Student [244] und Sahm [245], die bereits darauf hingewiesen hatten, dass die Dynamik von Erkrankungsprozessen in PV nicht genügend berücksichtigt wird. PV scheinen damit wenig hilfreich zur Eingrenzung von Behandlungsunsicherheit zu sein; bzw. sind sie für den untersuchten Kontext nur dann eine Hilfe, wenn der Patient*innenwille mit der ärztlichen Einschätzung (fachlich und moralisch) übereinstimmt. Das betrifft nicht nur Festlegungen zur Beendigung medizinischer Maßnahmen, sondern auch deren Einforderung. Ein diskrepantes Therapieziel und der Wunsch von Patientinnen nach Maximaltherapie finden sich auch bei Winkler et al. als Einflussfaktoren auf die Einbeziehung von Patient*innen bei Entscheidungen am Lebensende [246]. Die Ergebnisse belegen zudem die Hypothese von Hartog et al., die bei den formulierten Anwendungsbedingungen einer PV Interpretationsspielraum sehen und auf ärztliche Unsicherheiten über die Prognose als Grund für die Nicht-Einhaltung der Behandlungspräferenzen von Patient*innen hinweisen [240]. Und selbst wenn grundsätzlich ein palliatives Therapieziel formuliert wird, verdeutlicht die Palliativ-Dimension den breiten Interpretations- und Handlungsspielraum (vgl. Kap. 5.1.5.1.). Hier zeigt sich eine neue Herausforderung vor allem für die Behandlung onkologischer Erkrankungen, welche mit modernen Therapien auch in palliativen Situationen eine Lebensverlängerung bei guter Therapieverträglichkeit und damit Lebensqualität ermöglichen und damit das Feld der prognostischen Ungewissheit über den Todeszeitpunkt vergrößern [391]. 
Aus Vorarbeiten und eigener klinischer Erfahrung hatte ich als Präkonzept abgeleitet, dass Stationsärzt*innen im arbeitsteiligen und hierarchischen System Krankenhaus eine Schlüsselrolle für die Verbesserung der Versorgung Schwerstkranker und Sterbender einnehmen. Die Untersuchungsergebnisse legen nahe, dass zukünftige Forschung zusätzlich die primär hierarchische Entscheidungssituation, das individuelle Engagement des medizinischen Personals sowie die Eigenschaft des Krankenhauses als Lernort in den Blick nehmen muss, um die Bedeutung dieser Faktoren für die Integration der palliativen Perspektive auf Normal- und Intensivstationen adäquat zu erfassen.

\subsection{2 Ärztliche Einforderung eines Sterbendürfens im Krankenhaus: offene Bewusstheit oder (neue) Eindeutigkeit in der Behandlung?}

Die deutlichen Befunde über eine ärztliche Entscheidungshoheit in der medizinischen Behandlung konzentrieren sich für den Untersuchungskontext der ärztlichen Erfahrung mit der Behandlung Schwerstkranker und Sterbender in der empirisch fundierten Schlüsselkategorie: Das ärztliche Postulat vom Sterbendürfen im Krankenhaus kann als ein Antwortschritt in der Entscheidungskette interpretiert werden (vgl. Kap. 5.4.). Mit dem Begriff „dürfen“ rückt die rechtliche Regelung in den Blick. Die rechtliche Klärung der Unterscheidung von aktiver oder passiver Sterbehilfe und Sterbebegleitung zeigt sich jedoch nur als nachrangige Begründung für die Forderung nach einer Entscheidung. Deutlicher tritt mit der Wortwahl „dürfen“ die Entscheidungs- und Machtstruktur, die sich in das hierarchische System Krankenhaus eingeschrieben hat, in Erscheinung. Dies habe ich im vorangegangenen Kapitel diskutiert. Im Aufeinandertreffen der normativen Bilder im Postulat mit dem Klinikalltag werden nun Widersprüche, Konflikte und Unsicherheiten ebenso deutlich wie individuelle, informell-kollektive und strukturelle Handlungsspielräume.

Wie von Dreßke für Hospize beschrieben [119], zeichnen die interviewten Ärzt ${ }^{\star}$ innen auch für das Krankenhaus ein Idealbild des friedlichen Sterbens mit Zeit für Sterbebegleitung. Aber anders als ein Hospiz stellen sie das Krankenhaus nicht als „Labor des guten Sterbens“ [119, S. 14] mit einer Spezialisierung auf das Sterben bzw. einem Fokus auf natürliches Sterben dar. Die Frage nach der Natürlichkeit des Sterbens in Zeiten zunehmender medizinisch-technischer Möglichkeiten, im Kontext juristischer Überlegungen und der Finanzierung im deutschen Gesundheitswesen muss eher als ethisch-moralische Kategorie diskutiert werden [4, 117]. Die Nichteinmischung in einen Sterbeprozess ohne ein zeitlich bestimmbares Ende entspricht nicht der Behandlungsrealität eines Krankenhauses mit seinen effektivierten Liegezeiten und der Akut- und Heilungslogik. Das hat Konsequenzen für die Behandlung: Ein natürliches Sterben - idealisiert als ungestörtes und friedliches Sterben [119, S. 225ff] - ist im Krankenhaus weder strukturell und organisatorisch noch habituell vorgesehen. Die Charta zur Versorgung schwerstkranker und sterbender Menschen, die von beiden untersuchten Kliniken unterzeichnet wurde, ist keinem der IP be- 
kannt. Die vorgefundenen ärztlichen Ideale einer würdevollen Sterbebegleitung mit Zeit, Raum und Gespräch zeigen zwar eine gewisse Passung zu den Leitsätzen der Charta, die Klinikressourcen und Arbeitsorganisation jedoch nicht. Mit dem Zusatzentgelt für die Palliativkomplexbehandlung für jede Krankenhausstation und dem Zusatzentgelt für einen Palliativkonsildienst (PKD) ist ein finanzieller Rahmen geschaffen worden, doch trotz verbesserter Finanzierungsmöglichkeiten, die im Untersuchungszeitraum bereits existierten, zeigt sich keine veränderte bzw. verbesserte personelle oder räumliche Situation auf den Normalstationen (vgl. Kap. 5.1.2.).

Vogd konstatiert in einer soziologischen Rahmung der Behandlung im Krankenhaus: „Die Interferenz von medizinischer und administrativer Rationalität verhindert hier im Sinn von Glaser und Strauss einen > offenen Bewusstheitskontext $<$ und erzeugt den Angehörigen gegenüber einen > geschlossenen Bewusstheitskontext < . “ [382, S. 35]. Konnte Vogd in seiner Untersuchung auf verschiedenen Stationen eines Allgemeinkrankenhauses vor allem eine notwendige Aufrechterhaltung der Diffusität in der ärztlichen Behandlung Sterbender und in der Kommunikation mit Angehörigen als Handlungsspielraum (auch gegenüber den Krankenkassen) skizzieren [13, S. 348], verweisen die vorliegenden Befunde auf ethisch-moralische Konflikte der interviewten Ärzt`innen durch ebendiese diffuse Behandlung sowie auf das spannende Phänomen eines neuen Handlungsspielraumes gerade durch Konturierung und bewusste Festlegung. Inwieweit es sich bei der ärztlichen Einforderung eines Sterbendürfens im Krankenhaus um eine offene Bewusstheit in der Behandlung oder aber um eine (neue) Eindeutigkeit - und damit wieder geschlossene Bewusstheit - handelt, werde ich nachfolgend diskutieren.

In ihrer aktuellen Untersuchung kritisieren Saake et al. das „Paradigma vom bewussten Sterben“ [5], welches sie auf zwei deutschen Palliativstationen identifiziert haben, ähnlich wie Dreßke in Hospizen [119]. Seit der Studie von Glaser und Strauss [172] aus dem Jahr 1974 habe sich die 'geschlossene Bewusstheit' gewandelt zu einer inzwischen 'normativen Bewusstheit', verbunden mit der Erwartung, dass Patient*innen die Sterberolle einnehmen, so Saake et al. [5]. Auf den von ihnen untersuchten Palliativstationen werden „Ärzte sichtbar, die ihre Arbeit gut machen wollen, die aber mit der mangelnden - wenn man so sagen will - Sterbeakzeptanz ihrer Patienten kämpfen“ [5, S. 33]. Die Befunde der vorliegenden Arbeit mit dem Untersuchungskontext von Normal- und Intensivstationen zweier Akutkrankenhäuser mit 13 beteiligten medizinischen Fachdisziplinen zeichnen ein anderes Bild: Glasers und Strauss' Analyse eines geschlossenen Bewusstheitskontextes über Sterbesituationen erscheint so aktuell wie vor 45 Jahren. Darauf verweisen nicht nur die ärztlichen Aufklärungsstile (vgl. Kap. 5.2.4.), sondern auch die Festlegung der Heilung als zentralen Behandlungsauftrag (vgl. Kap. 5.1.3.). Was nun zutage tritt, ist ein moralisches und psychisches Unwohlsein in der erlebten Behandlung Sterbender. Die Ergebnisse verdeutlichen, dass es sich nicht nur um fehlende individuelle Bewältigungsressourcen oder eine Demoralisierung der Stationsärzt*innen handelt, sondern zudem um fehlende strukturelle und kollektive Ressourcen [295]. Insbesondere wenn Sterben 
als Misserfolg des ärztlichen Handelns gerahmt wird und die Kompetenzerwartung ausschließlich als Heilungserwartung existiert, führt das zu Abwehrmaßnahmen und Bedeutungsänderungen. Dies zeigt sich sowohl im vorgefundenen begrifflichen Paradox einer formal kurativen Behandlung als auch im Paradox der Nicht-Nutzung eines PKD trotz formuliertem Bedarf sowie in der Aus- oder Zwischenlagerung Sterbender.

Das Postulat erscheint damit weniger als eine Anforderung an Patient*innen, ihre Sterberolle anzunehmen. Vielmehr werden im Untersuchungskontext Ärztinnen sichtbar, die um die Anerkennung einer Versorgungsform ringen, die im Krankenhaus längst stattfindet. Die Sprechrichtung, die „kommunikative Verflüssigung“ [5, S. 49] über das Thema Sterben, die sich im Postulat zeigt, zielt also nicht auf Patient*innen und Angehörige, sondern ist intraprofessionell, interhierarchisch und organisational. In Anknüpfung an Vogds Analyse von „Täuschungsmomenten“ in der Behandlung palliativer und sterbender Patient*innen im Krankenhaus [13, S. 339ff] interpretiere ich die (stations)ärztliche Einforderung einer Entscheidung über das Sterbendürfen als Wunsch, in der Behandlung und ggf. auch in der Ärztin-Patient*in-Kommunikation nicht mehr täuschen zu müssen. Aber anders als Dörner in seinen beziehungsethischen Überlegungen [28] oder Lown mit einer Anleitung für das Umdenken in der Ärztin-Patient*in-Beziehung werben [46], verstehe ich die ärztliche Einforderung einer offenen Bewusstheit über das Sterben hier nicht als Wiederentdeckung oder Annahme der Sterbebegleitung als ärztlichen Behandlungsauftrag. Das Postulat bewegt sich in der Logik der dargestellten hierarchischen Entscheidung und richtet sich an Ober- und Chefärzt*innen, aber auch an den Gesetzgeber und die Politik. Damit beinhaltet der Wunsch nach Beendigung der Täuschung sowohl eine Absicherungsstrategie als auch eine Verantwortungsverschiebung. Beide Strategien bewahren die ärztliche Integrität. Die interviewten Stationsärzt*innen kritisieren fehlende Entscheidungen, kaum aber die Hierarchien, die den Entscheidungsprozess dominieren und/oder verzögern. Wie die Daten zeigen, wird im Gegenteil - gerade bei EoL-Entscheidungen - ein hierarchisches Entscheiden eingefordert. In allen Formulierungen wird die Entscheidungsverantwortung in der (medizinischen) Grenzsituation Sterben außerhalb der eigenen Person gesehen: in der Gesellschaft, im Gesundheitswesen und in der Politik, die Ressourcen zur besseren Versorgung bereitstellen müssen.

Verstehe ich das normative ethische und soziale Einfordern eines Sterbendürfens (und im Anschluss eine Sterbebegleitung) als Konsequenz und als einen neuen medizinischen Auftrag im Krankenhaus, so tritt die gegenwärtige Parallelität der Akutbehandlung und Palliativmedizin deutlich zutage. Eine Entscheidung, Erlaubnis und damit Beauftragung zur Sterbebegleitung erscheint somit zwar als ein Ausweg aus der bisherigen Täuschung in der Behandlung [13] sowie als ein Ausstieg aus dem 'Arrangement der Hoffnung', welches die an der Behandlung beteiligten Akteure bisher eingegangen sind [116], führt aber nicht zwangsläufig zu einer Aufhebung der Dichotomie der Behandlungslogiken. Auch mit einer Entscheidung für ein Sterbendürfen 
wird die Akut- und Heilungslogik nicht grundlegend erschüttert, sondern sie stabilisiert sogar die duale Betrachtung der Behandlung. Um beim primären Rahmen eines Krankenhauses zu bleiben, der „einen sonst sinnlosen Aspekt der Szene zu etwas Sinnvollem macht“ [212, S. 31]: Das Primat der Heilung erfordert auch bei Sterbenden intensivmedizinische und maximaltherapeutische Maßnahmen. Dieses Anliegen sehen die Ärzt`innen durch Forderungen von Patient`innen und Angehörigen, die mit einem Heilungsauftrag sogar im Sterbeprozess zur Behandlung im Krankenhaus erscheinen, vielfach bestätigt.

Die Sinnhaftigkeit dieser Maßnahmen zeigt sich in der ärztlichen Praxis jedoch erschüttert, wie ich entlang des ärztlichen Widerspruchserlebens der normativen Bilder im Postulat vom Sterbendürfen verdeutlicht habe (vgl. Kap. 5.4.). Die Vorstellung vom Krankenhaus als immerwährendem Ort der Lebensrettung und Heilung wird dekonstruiert, was sich auf die ärztliche Identität sowie das von Ärztinnen, Patientinnen und Angehörigen geteilte 'Arrangement der Hoffnung' auswirkt.

In der einleitenden Vorstellung der Rahmenbedingungen und des Forschungsstandes hatte ich bereits Eingrenzungsversuche der Ungewissheiten in der medizinischen Behandlung bei komplexen Problemlagen vorgestellt, wie medizinische Leitlinien, die surprise question und statistische Überlebenswahrscheinlichkeiten (vgl. Kap. 2.1.4.2.). Das ärztliche Postulat vom Sterbendürfen schafft nach dieser Lesart als Absicherungsstrategie eine neue medizinische Handlungsmöglichkeit durch die Herstellung einer eindeutigen Situation. Damit ist es eher Ausdruck einer fehlenden Ambiguität: Die interviewten Ärztinnen und Ärzte suchen mit dem Postulat eine eindeutige Lösung in einer widersprüchlichen Situation. Eine geringe bis fehlende Ambiguitätstoleranz kann dem gesamten klinischen Feld attestiert werden in der Suche nach eindeutigen Entscheidungen, die in akuten Situationen im Wortsinn überlebenswichtig sind. Gleichzeitig erscheint das Postulat vom Sterbendürfen als Lösungsversuch im Sinne einer Entlastung oder dialektischen Öffnung des ärztlich erlebten Widerspruchs von normativen Idealbildern und rekonstruierter Praxis im Krankenhaus.

Der von Antonovsky [280] als notwendig beschriebene Kohärenzsinn zur Gesunderhaltung von Menschen in prekären Situationen ist eine passende Rahmung für die vorliegenden, zunächst paradox wirkenden Befunde. Erst durch die Sinnhaftigkeit, Verstehbarkeit und Handhabbarkeit einer Situation, hier konkret die Behandlung Sterbender, erscheint sie für die Ärzt*innen kohärent. Als Suche nach Kohärenz lässt sich das Postulat auch als gleichzeitige Bewusstheit über sich widersprechende Gewissheiten im gleichen Kontext verstehen: einerseits die Gewissheit über den Akutbehandlungs- und Heilungsauftrag im Krankenhaus und andererseits die Gewissheit über die Erfahrung von Sterbenden. Diese Lesart erweitert die Perspektive, da sie das vielfach beschriebene Dilemma einer ärztlichen offenen Bewusstheit über Sterbesituationen in akuten Behandlungssituationen aufgreift. Das psychologische Konzept einer gleichzeitigen Bewusstheit, einer Double Awareness, wurde bisher vorrangig in Bezug auf Patient*innen diskutiert [227-228]. Ob die Gleichzeitigkeit der gegensätzlichen Behandlungsperspektiven im Krankenhaus als Ort der (erwarteten/ 
erhofften) Lebensrettung und als Ort des würdevollen Sterbens aushaltbar ist, stellt eine Frage neuer Praxisforschung dar.

Mit Blick auf die vorgestellten Dilemma-Situationen in der Begegnung mit Schwerstkranken und Sterbenden im Krankenhaus ist das ärztliche Postulat des Sterbendürfens Frage und Forderung zugleich und verweist auf Lernfelder mit einem ganzen Spektrum an Fragen. Diese zielen auf folgende Ebenen:

- medizinisch-technische Möglichkeiten und ihre (ethischen) Begrenzungen,

- berufsrechtliche und ethisch-moralische Grundsätze als Arzt bzw. Ärztin,

- gesellschaftlicher Umgang mit Sterbenden,

- ärztliche und Professions-Hierarchien im Krankenhaus,

- strukturelle und organisatorische Möglichkeiten und Begrenzungen der Integration von Palliativversorgung im Krankenhaus,

- intersektorale und sektorenübergreifende Zusammenarbeit,

- Finanzierung palliativmedizinischer und pflegerischer Maßnahmen,

- Patient*innenrecht bei der Einforderung der Durchführung oder Beendigung von Therapien,

- psychische Be- und Entlastung im Umgang mit Uneindeutigkeit, mit medizinisch-technischer Hilflosigkeit, mit Sterbenden und Sterbebildern, mit der als zunehmend erlebten kommunikativen Anforderung im Kontakt mit Angehörigen.

Als ein Präkonzept hatte ich formuliert, dass Behandlungsentscheidungen von Ärzt*innen bei palliativen Patient*innen und bei Sterbenden im Krankenhaus auf diffusem rechtlichen, fachlichen und organisatorischen Wissen basieren. Auf Grundlage der vorliegenden Befunde lässt sich hinzufügen: Mit der Einforderung eines Sterbendürfens im Krankenhaus als intraprofessionelle Bewusstheit über die Ambiguität in der medizinischen Behandlung werden das ärztliche Ethos herausgefordert und - als organisatorisch-ökonomische Bewusstheit - das gesellschaftliche Ethos über den Behandlungsauftrag eines Krankenhauses. Liest man die Entscheidung über das Sterbendürfen als neue Eindeutigkeit, wird die Dualität von Akut- und Palliativmedizin wiederhergestellt.

\subsubsection{Die Dualität von Akut- und Palliativmedizin im Krankenhaus}

Die Untersuchungsergebnisse weisen auf ein zentrales Missverständnis zwischen Palliativ- und Akutmedizin hin und bestätigen beschriebene Kulturunterschiede im deutschen Gesundheitswesen [24-25]. Das Missverständnis zeigt sich in der differenten Bezugnahme auf Qualität: Eine palliativmedizinische Perspektive definiert vorrangig Symptomlinderung als Behandlungsqualität [129]. Im Akutkrankenhaus ist das Nicht-Sterben eines Patienten/einer Patientin, d. h. die Lebensrettung und damit -verlängerung, vorrangiger Behandlungsauftrag und somit eine Behandlungsqualität. Die Analyseergebnisse verdeutlichen, dass die interviewten Ärztinnen jede 
Zustandsverschlechterung zunächst im Sinne der Akut- und Heilungslogik behandeln (vgl. Kap. 5.1.3.1.). Vor allem bei hämato-onkologischen Erkrankungen erfolgt sogar eine Erweiterung dieser Logik durch eine Rahmung als formal kurative Behandlung (vgl. Kap. 5.1.3.2.), und sie zeigt sich übergreifend im medizinischen Enthusiasmus begründet (vgl. Kap. 5.1.3.3.). Die vorgefundenen Behandlungsperspektiven legitimieren maximal- und intensivmedizinische Interventionen auch bei Schwerstkranken und Sterbenden. Den zentralen Behandlungsauftrag verdeutlichen die Qualitätsberichte der untersuchten Krankenhäuser [393-394], in denen Mortalität als Qualitätsindikator [81, S. 15] berücksichtigt wird. Auch die ärztlichen Wahrnehmungen von Patient*innen und Angehörigen, die eine maximaltherapeutische und intensivmedizinische Behandlung entgegen der medizinischen Indikation einfordern (vgl. Kap. 5.2.5.3.), sowie im Sterbeprozess befindliche Patient*innen, die zur Behandlung ins Krankenhaus kommen/gebracht werden (vgl. Kap. 5.3.2.), verweisen auf Lebensrettung/-verlängerung als zentralen Behandlungsauftrag. Eine Integration von PC in die medizinische Behandlung, wie sie von der WHO definiert [395], von Irwin \& van Gunten konzipiert [153] und von Temel als effektiv - sogar im Sinne der quantitativen Lebenszeitverlängerung - nachgewiesen wurde [154], ist rar im untersuchten Kontext. Auch bei nicht-sterbenden, sondern schwerstkranken Patient*innen im Sinne der Palliativ-Dimension findet eine Gegenüberstellung einer kausalen Behandlung der Erkrankung und einer „rein“ supportiven Behandlung bei Schwerstkranken und Sterbenden durch die interviewten Ärzt`innen statt. In der vorgestellten Änderung des Therapieziels im Behandlungsverlauf wird die Dualität der Behandlungskonzepte besonders sichtbar (vgl. Kap. 5.2.2.). Für die Beantwortung der Frage, warum die Integration palliativer Behandlungsansätze in das akute und kurative stationäre Setting so hürdenreich ist, bieten die vorliegenden Befunde vielfältige Begründungen. Vor allem das Paradox der Nicht-Nutzung und der Skepsis gegenüber einem PKD trotz formuliertem Unterstützungsbedarf ist erhellend (vgl. Kap. 5.3.4.). Gründe für die Nicht-Nutzung zeigen sich auf den Ebenen von Klinikorganisation, ärztlichem Habitus, Beziehungsethos, palliativmedizinischen Kenntnissen sowie eines begrifflichen Stigmas von 'palliativ'. Mit der Begrifflichkeit 'palliativ' und dementsprechend auch mit PKD wird von den IP v. a. ein Angebot für Sterbende assoziiert. Damit bestätigen sich für deutsche Krankenhäuser hinsichtlich aller untersuchten medizinischen Fachdisziplinen und -bereiche die Ergebnisse aus US-amerikanischen Kliniken [155, 309-311]: Palliative Angebote werden aufgrund der Gleichsetzung einer palliativen Behandlung mit Sterbebegleitung spät und häufig nicht einbezogen. Ganz eindeutig verweisen die Ergebnisse auf eine notwendige Klärung von Begriffen. Ob die Begriffsänderung von 'palliativ' zu 'supportiv', wie sie von medizinischen Fachgesellschaften inzwischen auch in Deutschland diskutiert wird [396], eine Nebelkerze ist oder ob damit tatsächlich eine Möglichkeit geschaffen wird, das Stigma einer Palliativbehandlung [397] oder die „Palliphobie“, wie Bruera es nannte [261], aufzulösen, bleibt eine Aufgabe zukünftiger Praxisforschung bzw. Weiterbildung. In diesem Zusammenhang stellt sich die Frage, ob es neben einer neuen begrifflichen Rahmung von Palliative 
Care nicht vielmehr eine „grundlegende Veränderung in der Art und Weise, wie Palliative Care praktiziert und dargestellt wird“ als gesamtgesellschaftliche Bildungsarbeit braucht, um den gegenwärtigen Dualismus der Behandlungsperspektiven aufzuweichen, wie Zimmermann und Kollegen im Anschluss an ihre Forschungsergebnisse im Kontext des kanadischen Gesundheitssystems fordern [397, E226].

In der Behandlung onkologischer Patient`innen zeigt sich am deutlichsten die Tendenz, eine palliative Perspektive in den Behandlungsverlauf einzubeziehen. Die IP greifen bei onkologischen Erkrankungen trotz der Ungewissheit des Verlaufs auf prognostische Kennzeichen wie die statistische Überlebenswahrscheinlichkeit zurück sowie auf Erfahrungen mit belastenden und symptomreichen Sterbebildern (vgl Kap. 5.1.5.2.2.). Um bei der Quantität zu bleiben: Auch die schnelle und einfache Erreichbarkeit eines $\mathrm{PKD}$ erweist sich als Kriterium für eine positive Wahrnehmung. $\mathrm{Ob}$ das palliativmedizinische Angebot von den Ärzt*innen überhaupt genutzt wird, hängt von ihren Vorerfahrungen, von der Kenntnis des Angebots, der Wahrnehmung der eigenen ärztlichen Verantwortung in der Beziehung zum Patienten/zur Patientin, der individuellen Einstellung zu und vom Wissen um PC sowie von der Abteilungskultur ab (vgl. Kap. 5.3.4.). Diese Einflusskriterien bestätigen Untersuchungen aus dem angloamerikanischen Raum [312-314]. Aufschlussreich ist, dass sich auch bei der Formulierung eines Unterstützungsbedarfs in der vorliegenden Untersuchung ein eher quantitatives Interesse im Sinne einer schnellen Verlegung sowie der Wunsch nach Übernahme nicht zu bewältigender kommunikativer oder anderer Aufgaben durch den PKD zeigt. Gleiches wird im übrigen auch hinsichtlich der Wahrnehmung einer Palliativstation deutlich: Die Mehrzahl der interviewten Ärzt*innen formuliert sowohl das Bild einer Sterbestation als auch den Wunsch nach einer schnellen Übernahme Sterbender (vgl. Kap. 5.3.3.4.). Die Befürchtung von Dunlop und Hockney [315], dass Palliativstationen zur Auslagerung des Sterbens führen, lässt sich auf Grundlage der vorliegenden Untersuchungsergebnisse teilen. Darüber hinaus bestätigt sich ihre Annahme, dass der Verlegungswunsch vorrangig mit einer häufig fehlenden palliativen Perspektive auf den primär behandelnden Stationen eines Krankenhauses einhergeht.

Im Kontext des Krankenhauses zeigt sich in der Dualität der palliativmedizinischen und der Akutbzw. heilenden Behandlungsperspektive eine fehlende Ambiguitätstoleranz. Die Behandlung Sterbender wird von den Ärzt*innen ethisch-moralisch gerahmt und die Akutmedizin mit einer Heilungsperspektive als primär angesehen. Die ärztlich vorgenommenen Rahmungen lassen sich als soziale Repräsentationen einer gesellschaftlich, gesundheitspolitisch, rechtlich und ethisch geführten Debatte über das Lebensende verstehen. 


\subsubsection{Fehlversorgung Schwerstkranker und Sterbender im Krankenhaus}

Insgesamt bestätigen die Untersuchungsergebnisse vielfältig die Fehlversorgung Sterbender im Krankenhaus - sowohl als palliativmedizinische Unter- als auch als kurative Überversorgung [49, 70, 149]. Eine interviewte Ärztin benennt die Fehlversorgung Schwerstkranker und Sterbender prägnant als Leerstelle: „Diese Patienten gehen da unter.“(AB02/663). Die Befunde der vorliegenden Studie ermöglichen Begründungen für diese Versorgungsleerstelle im Krankenhaus, die ich nachfolgend zusammenfasse.

Wie die in Kapitel 5.1. dargestellten Arbeitsbedingungen zeigen, sind die Folgen einer ökonomisch orientierten Krankenbehandlung mit Einfluss auf strukturelle und personelle Ressourcen in beiden untersuchten Forschungsfeldern deutlich erkennbar. Den bekannten Trend der Arbeitsverdichtung durch die Zunahme von Behandlungsfällen bei gleichzeitig verkürzter Liegezeit [83] aufgrund des FallpauschalenFinanzierungssystems beschreiben die IP als „Durchlaufmedizin“ und „Arbeit im Akkord“. Insbesondere wirkt sich der Mangel an Pflegekräften negativ auf die gesamte Patient*innenversorgung aus. Speziell bei symptombelasteten (und sterbenden) Patient ${ }^{\star}$ innen bleibt im arbeitsverdichteten Alltag die notwendige Verlaufskontrolle für eine adäquate symptomatische Behandlung aus.

Im vorgefundenen Ideal einer würdevollen Sterbebegleitung durch gute Pflege zeigt sich der von Findeiß skizzierte „Mythos der Ganzheitlichkeit der Pflege“ [88, S. 307] als nach wie vor wirksam, denn die interviewten Ärzt*innen schreiben die Versorgung dieser Patient*innengruppen sowie insgesamt eine patient*innennahe Arbeit vorrangig den Pflegekräften zu (vgl. Kap. 5.1.5.2.). Bei einem Pflegekräftemangel wird das Ideal gestört und damit die ärztliche Behandlungszufriedenheit.

Arbeitsverdichtung und Personalmangel verstärken zudem vor allem interprofessionelle Kommunikations-, Interaktions- und Kooperationsdefizite zwischen Ärztinnen und Pflegekräften, die hierarchisch und strukturell bereits existieren. Wird die Versorgung Schwerstkranker und Sterbender vor allem als pflegerische Aufgabe verstanden, zeigt sich hier eine wichtige Begründung für den Versorgungsmissstand: Aufgrund der fehlenden Überschneidungsräume und -zeiten (z. B. interprofessionelle Übergaben, gemeinsame Visiten) gibt es eine mangelnde Rückkopplung der pflegerischen Patient^innenbeobachtung an Ärztinnen (vgl. Kap. 5.1.2.3.) sowie eine mangelnde intra- und interprofessionelle Verständigung über eine Therapiezieländerung bei einem verschlechterten Erkrankungszustand (vgl. Kap. 5.2.2.). Die somit diffuse Situation birgt Konfliktpotential bei diskrepanten Einschätzungen und ist Ausgangspunkt einer Fehlversorgungskette, die $u$. U. in eine ausbleibende adäquate Behandlung mündet (vgl. Kap. 5.2.1.).

Das ärztliche Ideal der Zeit für Gespräche mit Patient`innen und Angehörigen sowie ein ärztliches Beziehungsethos treffen in der routinierten und verdichteten Praxis auf kommunikative Erfordernisse, v. a. mit den Angehörigen Schwerstkranker und Sterbender, die als zunehmend erlebt werden. Diese Gesprächs- und Organisati- 
onsarbeit in der Begleitung von Sterbesituationen ist (ohne DRG-Finanzierung) organisatorisch weder als Arbeitszeit eingeplant noch habituell anerkannt (vgl. Kap. 5.2.4.). Der Missstand wird vor allem mit individuellem Engagement der Stationsärzt`innen und informeller (interdisziplinärer und interprofessioneller) Kooperation gelöst. Die individuellen Lösungen betreffen sowohl die Kommunikation mit Angehörigen als auch die Überleitung in den pflegerischen Kompetenzbereich. Beides macht eine würdevolle Sterbebegleitung im Sinne der ärztlich formulierten Ideale möglich, z. B. durch die Verlegung in ein Einzelzimmer oder die Förderung der Anwesenheit von Angehörigen (vgl. Kap. 5.1.5.2.3.). Die Anerkennung dieser Arbeit innerhalb des interprofessionellen Teams, durch ärztliche Kolleg*innen und direkte Vorgesetzte reicht von unterstützend bis zu einem völligen Gratifikationsdefizit (vgl. Kap. 5.1.2.1.). In der knappen Zeit wird somit die kommunikative Anforderung zur stärksten Belastung in der ärztlichen Konfrontation mit Sterbenden, was bisherige wissenschaftliche Befunde zu ärztlichen Einstellungen in der Sterbebegleitung bestätigt [36] (vgl. Kap. 2.2.3.).

Die vorgestellten Behandlungsaufträge im Arbeitskontext zeigen eindrücklich die habituelle Verfestigung des Heilungsauftrags: Schwer Kranke und potentiell Sterbende im Krankenhaus stören und verunsichern den ärztlichen und medizinischen Enthusiasmus. Gerade in verunsichernden und uneindeutigen bzw. diffusen Situationen wird in den Ergebnissen die Stärke medizinischer Mythen deutlich [398]. Zugleich werden Widersprüche zur Behandlungspraxis sichtbar:

- Der Mythos der medizinischen Eindeutigkeit trifft in der klinischen Arbeit auf uneindeutige, „schwammige Situationen“(AB11/403-405).

- Der Mythos des ärztlichen Heilungsvermögens, das „leicht größenwahnsinnige Anliegen, das dann immer hinzukriegen“ (AB21/288-279), trifft auf das Erleben von nicht heilbar oder sterbenden Patient*innen.

- Der Mythos der medizinisch-technischen Machbarkeit kollidiert mit der Sinnfrage und dem ärztlichen Ethos: „Ist DAS jetzt überhaupt noch das Richtige, was wir tun hier für den Patienten?“ (AB18/201).

Die größte ärztliche Herausforderung besteht darin $\mathrm{zu}$ akzeptieren, dass medizinische Behandlungen und ärztliche Handlungen nicht immer heilen können. Wenn die Perspektive der Akuität und Heilung fehlt, wird für die kleine Patient*innengruppe der Sterbenden im Krankenhaus kein wesentlicher ärztlicher und medizinischer Behandlungsauftrag mehr gesehen. Die Ergebnisse zeigen im Anschluss an Herschbach einerseits eine ärztliche Verunsicherung über die Handlungsmöglichkeiten in der Konfrontation mit Sterbenden, vor allem bei Stationsärzt`innen mit hohem Arbeitsdruck [256]. Eingebettet in einen Arbeitskontext der Akut- und Therapiestationen ohne Fokus auf palliative Symptombehandlung bzw. mit zwangsläufig symptomauslösenden (z. B. Übelkeit, Fieber, Schmerzen) therapeutischen Maßnahmen (wie eine Operation oder Chemotherapie) benennen die IP vorrangig ethisch-moralische (z. B. terminale Sedierung) und rechtliche (z. B. Abgrenzung zur Sterbehilfe) 
Unsicherheiten. Andererseits formulieren vor allem die IP aus der Onkologie, der Anästhesie und den Intensivbereichen kaum medizinische Unsicherheit in der palliativen Symptombehandlung, die allerdings vor allem mit Schmerztherapie gleichgesetzt wird. Deshalb kann man bei der Fehlversorgung Sterbender nicht nur auf schlechte Ausstattung und administrative Probleme verweisen. Genauso relevant erscheint ein institutionell, medizinisch und habituell fehlender Behandlungsauftrag im Krankenhaus außerhalb einer Palliativstation. Berücksichtige ich die Lehrfunktion v. a. in komplexen Behandlungssituationen, die Ober- und Chefärztinnen als Vorbild und Mentor^in zugeschrieben wird, zeigt sich eine Lehr-Leerstelle als möglicher Ausgangspunkt für eine Fehlversorgung Schwerstkranker und Sterbender (vgl. Kap. 5.1.4.). Das Einbeziehen einer palliativmedizinischen Perspektive in die klinische Lehre und insgesamt in die Kultur der medizinischen Behandlung wird vom individuellen oberärztlichen/chefärztlichen Engagement bestimmt. Der palliativmedizinische Kenntnisstand von Ober- und Chefärzt`innen stand nicht im Fokus der Untersuchung, aber mit großer Wahrscheinlichkeit liegt ihr Studium länger als 5 Jahre zurück und Palliativmedizin war somit kein regelhafter Inhalt (vgl. Kap. 2.1.5.3.). Ohne Fort- und Weiterbildungen und individuelles Engagement muss ihr Wissen damit als ähnlich basal und von persönlichen Einstellungen beeinflusst angenommen werden, wie es in Bezug auf die Stationsärztinnen herausgearbeitet wurde. Vor dem Hintergrund der strukturellen Bedingungen mangelnder Austauschräume und einer hierarchischen Entscheidungskultur bleibt der Einfluss individueller Erfahrungen auf die Behandlungsperspektive meist unreflektiert.

Die vorrangige Heilungsperspektive wirkt sich auch auf die Priorisierung von kurativen vor palliativen Patient*innen in der ärztlichen Aufmerksamkeit aus (vgl. Kap. 5.3.1.). Ob dies auch einer ökonomischen Logik folgt, lässt sich entlang der Ergebnisse nicht klären. Deutlich wird die ökonomische Behandlungsorientierung jedoch in der Verlegungspraxis (vgl. Kap. 5.3.). Als Fehlversorgung lässt sie sich dann fassen, wenn Schwerstkranke oder Sterbende aufgrund von „Belegungsdruck“ verlegt oder „zwischengelagert“ werden, und dies ohne adäquate Weiterbehandlung oder kommunizierte Überleitung (vgl. Kap. 5.1.2.1.). Zusätzlich zum Verlegungsdruck erweist sich auch fehlendes Wissen über ambulante palliative Versorgungsstrukturen als ursächlich für diese Praxis (vgl. Kap. 5.3.3.5.). Fehlendes ärztliches Strukturwissen kann auch in eine Nicht-Verlegung Schwerstkranker und Sterbender münden. Auch wenn das Entlassungsmanagement von den IP als Aufgabenbereich des Sozialdienstes beschrieben wird, zeigt sich die Notwendigkeit einer ärztlichen Entscheidung über das Therapieziel bestätigt, um diesen Prozess in Gang zu setzen. Erfolgt eine Therapiezieländerung nicht oder spät bzw. wird sie nicht oder spät (interprofessionell) kommuniziert, kann das eine adäquate Verlegung und würdevolle Behandlung verhindern. In der Folge bleibt die Aufklärung von Patient*innen und/oder Angehörigen aus, wird $u$. U. der Patient*innenwille nicht berücksichtigt und die Weiterbehandlung erfolgt weiterhin in der Heilungslogik. Der Akutfokus im Krankenhaus unter den Bedingungen einer ökonomisierten Krankenbehandlung verhindert zudem 
eine vorausschauende Versorgungsplanung mit koordinierter Entlassungsplanung und sektorenübergreifender Kooperation (vgl. Kap. 2.1.3.).

Zusätzlich zu den beiden Kennzeichen ungenügendes Strukturwissen und Verlegungsdruck für eine Nicht-Verlegung als Fehlversorgung ist ein dritter Aspekt bedeutsam: Medizinische Komplikationen im stationären Behandlungsverlauf mit daran anschließend (dauerhaft) notwendigen intensiven medizinischen und pflegerischen Maßnahmen verhindern eine Entlassung oder Verlegung. Diese Patient*innen werden zu Langliegern, die prognostisch sowohl Sterbende als auch chronisch Kranke sein können, zunächst - und ohne Therapiezieländerung - aber Schwerstkranke mit einer unsicheren Erkrankungsperspektive sind und damit weiter in der kurativen Logik ohne die Einbeziehung palliativer Hilfen behandelt werden (vgl. 5.1.5.3.). Mit dieser medizinisch unsicheren Perspektive werden Langlieger als besondere ärztliche Herausforderung erlebt, da sie Auslöser für kurative Frustration sind, welche den medizinischen Enthusiasmus bremst und ethisch-moralische Fragen provoziert.

Die Ergebnisse zur Aufnahmepraxis der untersuchten Kliniken verweisen auf weitere aufschlussreiche Phänomene zur Klärung einer möglichen Fehlversorgung Sterbender: Zum einen erklärt das Beibehalten der akuten Behandlungslogik - nicht nur durch die Behandler, sondern ebenso durch Sterbende (und ihre Angehörigen) - die Beanspruchung der Rettungsstellen (vgl. Kap. 5.3.2.). Der Akutlogik folgend kommt es zu Verlegungen Sterbender von der Rettungsstelle auf eine ITS, wenn dies nicht explizit durch den Patienten/die Patientin abgelehnt wurde bzw. wenn ärztlich nicht ein Sterbendürfen entschieden wird (vgl. Kap. 5.4.). Zum anderen benennen die IP ein Triagesystem, in dem bekannte Patient*innen vor dem Hintergrund einer Abteilungsphilosophie, die Sterbebegleitung als Behandlungsethos für bekannte Patient*innen formuliert, auch als Sterbende aufgenommen werden. Inwieweit es sich um ein offen kommuniziertes Angebot handelt oder ob die Inanspruchnahme der Rettungsstelle durch Sterbende (vielfach in Begleitung Angehöriger) Folge einer vorab fehlenden ärztlichen Aufklärung und Überleitung in ambulante (spezialisierte) Palliativversorgungsstrukturen ist, bleibt offen. Vogd hat diese Situation für Allgemeinkrankenhäuser als ein eher unkommuniziertes „raffiniertes Arrangement der Herstellung eines diffusen Rahmens“ beschrieben [13, S. 364], um eine Sterbebegleitung organisatorisch möglich zu machen.

Die IP zeigten sich in den Interviews von der eingeführten Begriffspaarung 'schwerstkrank und sterbend', in Anlehnung an die „Charta zur Betreuung schwerstkranker und sterbender Menschen“ [1], irritiert bzw. lehnten diese Kombination ab. Ihre Reaktionen bergen eine wichtige Begründung für eine potentielle Fehlversorgung: Alle Patient*innen im Krankenhaus werden als schwer krank wahrgenommen und grundsätzlich dem Primat der Akuität und Heilung bzw. Lebensverlängerung folgend behandelt (vgl. Kap. 5.1.3.). Ohne eine Therapiezieländerung wird die Heilungslogik mit maximal- und intensivtherapeutischen Maßnahmen auch bei Sterbenden beibehalten, was eine mögliche Erklärung für ihre kurative Überversorgung ist. Die prognostische Einschätzung zeigt sich als zentrales Kriterium für eine Therapieziel- 
änderung (vgl. Kap. 5.2.2.). Im Konzept der formal kurativen Behandlung erfolgt beispielsweise eine begriffliche Erweiterung und damit sichere Rahmung einer hoch prekären Erkrankungs- und Behandlungssituation, um in der Heilungslogik ärztlich handlungsfähig zu bleiben (vgl. Kap. 5.1.3.2.).

Vor allem für onkologische Erkrankungen wird sehr deutlich zwischen palliativem und kurativem Behandlungsziel unterschieden. Für eine palliative Erkrankungssituation differenzieren die IP entlang zweier Kriterien: Prognose und Symptomlast der Patient*innen. In der Palliativ-Dimension wird die Spannweite der Einschätzungen sichtbar (vgl. Kap. 5.1.5.1.). Eine allgemeine palliative Erkrankungseinschätzung gibt somit zwar eine Gewissheit der Unheilbarkeit mit ungewissem Todeszeitpunkt [172], aber noch keine Auskunft über eine Begrenzung des therapeutischen Spielraums im Krankenhaus. Denn im Sinne der Akutlogik können alle medizinischen Interventionen auch ohne Heilungsauftrag erfolgen. Ein (frühzeitiges) Einbeziehen palliativer Hilfen verschiebt den Fokus auf die Lebensqualität, wie von der WHO definiert [129] und vor allem für die Onkologie differenziert beschrieben [153]. Dies wird vor dem dargestellten Hintergrund erschwert.

In der Zusammenschau aller benannten Fehlversorgungsmomente als komplexes Begründungsmuster auf individueller, sozialer, struktureller und administrativer Ebene wird deutlich, warum isolierte Vorschläge und Konzeptionen zur Verbesserung der Versorgungssituation im Krankenhaus fehlschlagen müssen.

\subsection{Methodologische Betrachtung}

Nachfolgend werde ich die Güte der vorliegenden sozialwissenschaftlichen Forschungsarbeit diskutieren. Stärken und Limitationen expliziere ich entlang spezifischer Gütekriterien für qualitative Untersuchungen [339-340, 343], konkret an den aktualisierten ansatzübergreifenden Standards für insgesamt heterogene Forschungsstrategien, die Stübing und Kolleg*innen 2018 formuliert haben [342].

Gegenstandsangemessenheit: Das qualitativ explorative Vorgehen dieser Studie hat sich mit der Methodentriangulation von Kontextanalyse, Interviews und Gruppendiskussion als methodischer Ansatz mit großem Erkenntnispotential erwiesen [343, S. 310], wie u. a. die Analyse der vielfältigen Begründungen für die Fehlversorgung schwer kranker und sterbender Patient*innen im Krankenhaus zeigt. Die Passung von Kontextanalyse und Interviews zur Untersuchung der Forschungsfragen war gegeben. Justierungen der Methoden wurden in der Gruppendiskussion und bei der Nutzung der Netzwerkkarte notwendig: Im Studienverlauf wurde erkennbar, dass die institutionellen Bedingungen so stark hierarchisch und gleichzeitig strukturell so prekär wirken, dass der partizipative Ansatz (im Diskussionsforum) mit eben jenen Herausforderungen kollidiert, vor denen Ärztinnen stehen und die zentrale Analyseergebnisse sind. Hier bestätigte sich die vorab formulierte Sorge einiger IP, offen Kri- 
tik an Kommunikations-, Entscheidungs- und Behandlungsstrukturen im Krankenhaus zu üben. Traf in den Einzelinterviews das Studiendesign auf große Akzeptanz, erwies sich die Gruppendiskussion als methodische Grenzerfahrung. Von Unger hat bereits auf die Herausforderungen für partizipative Forschung im medizinischen Feld hingewiesen [349, Abs. 75-77]. Im Sinne eines emanzipatorischen Ansatzes erscheint das Studiendesign dennoch fruchtbar und wurde von den interviewten Ärzt*innen als Anerkennung ihrer Erfahrungen wahrgenommen. Das Wissen um eine Veröffentlichung beschrieben sie als Partizipationsschritt.

Der Verzicht auf die egozentrierte Netzwerkkarte [354] im Projektverlauf entspricht einer gegenstandsangemessenen Methodenentwicklung. Methodisch ist dies folgendermaßen zu erklären: Den IP gelingt es, in der Erzählung bereits zusammenfassend ihre konkreten Arbeitsstrukturen und Ressourcen für die Versorgung schwerstkranker und sterbender Patient*innen darzustellen, und sie zeigen sich irritiert durch eine formale Abfrage im Anschluss an ihre Erzählung. Kann eine Netzwerkkarte der komplexen Erzählung gerecht werden? Die Abbildung eines Netzwerks allein über eine grafische Darstellung verleitet m. E. dazu, dieses Bild allzu leicht mit einer realen Struktur gleichzusetzen. Strategische, organisatorische oder habituelle Aspekte der Akteursinteraktionen innerhalb eines Netzwerkes lassen sich im Untersuchungsfeld nicht auf einer eindimensionalen Netzwerkkarte visualisieren.

Empirische Verankerung: Im eigenen Arbeitsfeld zu forschen birgt die Gefahr der Verzerrung, z. B. durch zu positive oder zu negative Darstellungen des ärztlichen Handelns, die Befangenheit oder auch ein Wunschdenken aufgrund meiner Teilhabe am klinischen Geschehen. Doch Bergold \& Zaumseil schlagen eine „langjährige Beheimatung“ der Forscherin im Feld sogar vor [399, S. 142]. Die lange Zeit des Eindenkens in das Praxisfeld und der Vertrauensaufbau zu den Akteuren müssen dann nicht während des Forschungsprozesses erreicht werden, sondern sind vorgelagert. Meine Kenntnisse über das Forschungsfeld Krankenhaus waren zudem hilfreich, um die wechselseitige Fremdheit von theoretischen Konzeptionen und klinischer Praxis zu überwinden. Die Reflexion meines spezifischen Zugangs oder besser meiner Doppelrolle im Feld, eine soziale Erwünschtheit in den Gesprächen mit den Forschungsteilnehmer*innen und meine Reaktivität habe ich in Kapitel 4 dargestellt und reflektiert. Im Rahmen der interdisziplinären Studiengruppe und weiterer Diskussionsgruppen, die den Datenerhebungs- und Auswertungsprozess begleiteten, konnte ich meine Interpretationen immer wieder hinterfragen. Auch ein zeitweiliger Rückzug aus dem klinischen Arbeits- und Forschungsfeld in der Phase der Kategorienbildung ermöglichte mir die notwendige Distanz. Die im zirkulären Prozess der Datensammlung und -auswertung erreichte theoretische Sättigung verweist auf eine starke empirische Verankerung der vorliegenden Untersuchung und bestätigt die gewählten Samplingstrategien. Alle Hypothesen und theoretischen Feststellungen sind mit empirischen Befunden belegt. Mit dem weiterentwickelten GTM Ansatz Situationsanalyse [324] ist zudem eine kontextuelle Verankerung gewährleistet. Eine kommunika- 
tive Validierung der Analyseergebnisse erfolgte im Diskussionsforum. Die Daten flossen wiederum in die anschließenden Analysen ein.

Theoretische Durchdringung: Strübing et al. benennen das Gütekriterium der theoretischen Durchdringung als komplementäres Kriterium zur empirischen Sättigung [342, S. 9]. Die Vielfalt der dargestellten Theoriebezüge in Kapitel 2 belegt noch nicht die Qualität, sondern erst der Hinweis auf die notwendige interdisziplinäre Betrachtung für die komplexe Forschungsfrage und das bereits vielfach wissenschaftlich bearbeitete Feld Krankenhaus. Aus dem klinischen Feld kommend, gelang es mir über die Rezeption der Theoriebezüge die notwendige theoretische Sensibilität [365, S. 50] und Irritation in der Wechselbewegung zwischen Datenerhebung und Datenauswertung aufrechtzuerhalten. Widersprüche in der gleichzeitigen Betrachtung der unterschiedlichen Theoriebezüge aus psychologischer, medizinethischer und medizinsoziologischer Forschung sowie die Reflexion empirischer Arbeiten zu einzelnen Fragestellungen der Arbeit, wie ärztliches Belastungserleben im Kontakt mit Schwerstkranken und Sterbenden oder Evaluation spezialisierter palliativmedizinischer Angebote, habe ich einleitend diskutiert und für die Weiterentwicklung der Fragestellung genutzt.

Intersubjektive Nachvollziehbarkeit: Mit der Dokumentation des Forschungsdesigns und Forschungsprozesses in Kapitel 4 habe ich mein Vorgehen transparent dargestellt. Alle verwendeten Materialien wie der Interviewleitfaden, die Aufklärungs- und Informationsbögen sowie die pseudonymisierten Interviews und Protokolle der Gruppendiskussion sind den Gutachter*innen zugänglich. Dem GTM Ansatz folgend, habe ich mein Vorwissen in Kapitel 3 mit der Weiterentwicklung der Fragestellung und den explizierten Präkonzepten ausformuliert. Die Kodierung und Kategorienbildung erfolgte im Sinne der GTM und wird im Ergebniskapitel kenntlich gemacht. Alle Deutungen, Hypothesen und Lesarten sind mit Zitaten oder anderen Quellen belegt. Durch die Etablierung einer Studiengruppe für die Dauer des gesamten Forschungsprozesses und mit Hilfe weiterer Arbeitsgruppen erfolgte eine prozessuale Validierung. Das Diskussionsforum nutzte ich als kommunikative Validierungsstrategie [340, S. 320]. Für Struktur und Gliederung der Arbeit habe ich zwar den klassischen Aufbau von Theorie, Empirie und Diskussion gewählt. Der rote Faden einer detaillierten Rekonstruktion vielfältigen Widersprucherlebens als Erkenntnisprozess zieht sich jedoch von den einleitenden Worten bis zum Ausblick.

Limitation: Die Ergebnisse formuliere ich für Akutkrankenhäuser im großstädtischen Raum und für medizinische Fachdisziplinen, die an der Behandlung Schwerkranker, vorrangig onkologisch Erkrankter, beteiligt sind, sowie insbesondere für die Maximalversorgung in einer Universitätsklinik. Auch wenn Ergebnisse an den Kontext gebunden bleiben und damit nur begrenzt verallgemeinerbar sind [339, S. 251], bietet Markard folgende Überlegung an: „Verallgemeinerungsmöglichkeiten liegen 
nicht in zentralen Tendenzen, sondern in der Herausarbeitung gesellschaftlich vermittelter Handlungsmöglichkeiten.“ [335, Abs. 31]. Mit einer dichten Situationsanalyse habe ich das ärztliche Erleben und Handeln als soziale Repräsentation gesellschaftlich vermittelter Handlungsmöglichkeiten herausgearbeitet. Die Beschränkung auf Ärzt^innen als IP kann als Einengung der Perspektivenvielfalt im Krankenhaus angesehen werden, in dem viele Professionen zusammen tätig sind. Die Fokussierung hat allerdings eine konsequente Rekonstruktion des ärztlichen Blicks ermöglicht. Zudem repräsentiert das stationsärztliche Handeln Novizen in der medizinischen Behandlung und organisatorischen Struktur eines Krankenhauses und macht damit in besonderer Weise gegenwärtige Praxis sichtbar [159]. In einer anschließenden Forschungsarbeit wäre die Prüfung der Ergebnisse für Interaktionsprozesse und damit eine Erweiterung der einbezogenen Perspektiven im Forschungsfeld wünschenswert und sicherlich gewinnbringend. Auch wenn die Daten im Zeitraum von 2013 bis 2014 erhoben wurden, sind die Befunde aktuell. Einzig der Palliativkonsildienst (PKD) erscheint inzwischen aufgrund einer fest verhandelten Finanzierung und damit besseren personellen Besetzung deutlich stärker implementiert und genutzt, zumindest in der untersuchten Universitätsklinik.

Relevanz und Originalität: Die Relevanz der Fragestellung und das bestehende Forschungsdesiderat habe ich ausführlich dargestellt. Mit der dichten Beschreibung der gegenwärtigen Behandlungssituation Schwerstkranker und Sterbender im Krankenhaus liefern die Ergebnisse komplexe Begründungsmuster und gleichzeitig Hinweise für ebendiese Praxis. Widersprüche in den Daten wurden in der Ergebnisdarstellung als Erkenntnismomente offengelegt, z. B. das Paradoxon der Nicht-Einbeziehung eines Palliativkonsildienstes. Im ärztlichen Postulat vom Sterbendürfen sind die Widersprüche als zentrale Merkmale des theoretischen Konstrukts gekennzeichnet. Damit schließen die Befunde bisherige Forschungslücken und führen den Nachweis, dass die dringend notwendige Theorie-Praxis-Verständigung gelingen kann.

\subsection{Ausblick}

Krankenhäuser sind und bleiben perspektivisch der häufigste Sterbeort in Deutschland, und der Tod wird mit der wachsenden Zahl chronisch verlaufender Erkrankungen immer seltener plötzlich eintreten. Damit bleibt auch die Frage nach Sterbebegleitung auf Normal- und Intensivstationen eines Krankenhauses ein Thema sowohl für alle Akteure und ihre sozialen Beziehungen als auch für die medizinische Behandlung und pflegerische Versorgung.

Als eine zentrale ärztliche Begründung für eine schlechte Versorgung schwerstkranker und sterbender Patient^innen im Krankenhaus habe ich den Pflegekräftemangel rekonstruiert. Dieser - nicht nur ethische - Missstand wird inzwischen gesundheitspolitisch und gesellschaftlich deutlich wahrnehmbar verhandelt. Mit dem 
Gesetz zur Stärkung des Pflegepersonals wurden zusätzliche Pflegekräfte für alle Versorgungssektoren einschließlich ihrer besseren Bezahlung und eine zusätzliche Finanzierung von Krankenhäusern beschlossen [400]. Die vorliegenden Befunde, die aus ärztlicher Sicht die Pflegenden als kompetenter im Erkennen von Sterbeprozessen und beauftragt mit der Sterbebegleitung zeigen, fordern darüber hinaus professionstheoretische Überlegungen heraus. Die Frage, wie sich die Umverteilung der Ressourcen im Gesundheitswesen und die angestrebte Aufwertung des Pflegeberufes - auch durch die Akademisierung der Pflege - auf das diffizile und (professions-) hierarchische Behandlungs- und Behandlergefüge im Krankenhaus auswirken werden, ist ein lohnender Ausgangspunkt nicht nur zukünftiger Forschung, sondern auch für die Praxisbegleitung. Zunehmend kritisch, jedoch nicht als konkrete Perspektive, wird die Abschaffung der DRG-Fianzierung für Krankenhäuser diskutiert, welche nachweislich die ökonomischen Zwänge der Krankenbehandlung insgesamt verschärft und auch in der Behandlung Sterbender zu Über- oder Unterversorgung führt - wie die Befunde dieser Untersuchung zur Verlegungspraxis zeigen.

Neben diesen Auswirkungen ökonomischer, struktureller und kultureller Bedingungen der Krankenbehandlung wird sich in den kommenden Jahren zeigen, wie und ob palliativmedizinisches Wissen, dessen Vermittlung erst seit 2014 in das Medizinstudium integriert ist, die Behandlung von palliativen und/oder sterbenden Patient*innen verändert. Das Krankenhaus ist und bleibt in jedem Fall ein wichtiger Lernort auch für nachfolgende Ärztinnengenerationen - eine hinreichende Anerkennung der Lernsituation Praxis steht noch aus und ist daher dringend geboten.

Das in der vorliegenden Untersuchung herausgearbeitete interdisziplinäre Missverständnis über das Behandlungsziel Lebensqualität mit Auswirkungen auf die kollegiale und sektorale Zusammenarbeit sowie auf die Ärztin-Patientin-Kommunikation muss in palliativmedizinischer Forschung und Praxis als Integrationshürde einer palliativen Perspektive in die Akutbehandlung konsequent berücksichtigt werden.

In den vorgelegten Befunden werden Prinzipien und Strukturen eines spezifischen ärztlichen Handelns im Kontakt mit Schwerstkranken und Sterbenden sichtbar. Damit liefert das ärztliche Postulat vom Sterbendürfen einen theoretischen Beitrag für ein weiter auszuarbeitendes Sterbedispositiv.

Das Postulat vom Sterbendürfen erweist sich in der Praxis darüber hinaus in bemerkenswerter Weise als prognostisches Instrument für individuelles und organisatorisches Handeln in der Begegnung mit Schwerstkranken. Es erscheint mir daher lohnend, das Postulat hinsichtlich Kommunikations- und Interaktionssituationen zwischen Behandlern, Patient*innen, Angehörigen und Organisationen weiter auszudifferenzieren und als Vermittlung zwischen Theorie und Praxis nutzbar zu machen. 
